\title{
Expression and prognostic significance of TRPV6 in the development and progression of pancreatic cancer
}

\author{
HE SONG, MING DONG, JIANPING ZHOU, WEIWEI SHENG, XIN LI and WEI GAO \\ Department of Gastrointestinal Surgery, The First Hospital of China Medical University, \\ Shenyang, Liaoning 110001, P.R. China
}

Received June 20, 2017; Accepted January 3, 2018

DOI: $10.3892 /$ or.2018.6216

\begin{abstract}
In this study, we aimed to clarify the expression and biological functions of TRPV6 in human pancreatic cancer (PC) tissue and pancreatic cancer (PC) cell lines. TRPV6 was up-regulated in the primary cancer tissues from pancreatic cancer (PC) patients. TRPV6 may regulate multiple proteins related to apoptosis, cell cycle and metastasis pathways. Silencing TRPV6 significantly inhibited invasion, proliferation and migration and induced apoptosis and cell cycle arrest. TRPV6 plays a promising role of an oncogene in pancreatic carcinogenesis and represents the new potential biomarker for PC.
\end{abstract}

\section{Introduction}

$\mathrm{PC}$ is one of the most aggressive and fatal cancers due to rapid metastasis, easy recurrence, early invasion and resistance to conventional chemotherapy. Survival rates of PC remained stagnant during the last half century (1). It was confirmatively suggested that the early detection of PC implied a better prognosis in the long-term survival (2). It is essential to search for novel biomarkers for early detection and to promote the efficiency of chemotherapy.

The TRPV6 channel is a calcium cation channel protein, which is the major constituent of superfamily of transient receptor potential (TRP) channels, further in terms of subfamily vanilloid member 6 , which regulates the calcium homeostasis in the epithelial tissue of human organs (3). TRPV6 is overexpressed in colon cancer, breast cancer, prostate cancer, parathyroid cancer and thyroid cancer (3-6), however decreased TRPV6 expression was observed in esophageal cancer, non-small cell lung cancer and renal cancer (7-9). Overexpression of TPPV6 in many cancers indicates the idea of this protein as encoded by a probable oncogene, but the level of TRPV6 in other cancers showed a tendency of a

Correspondence to: Dr Ming Dong, Department of Gastrointestinal Surgery, The First Hospital of China Medical University, Shenyang, Liaoning 110001, P.R. China

E-mail: dongming@cmu.edu.cn

Key words: TRPV6, pancreatic cancer, expression, progression tumor-suppressor. Nevertheless, the expression and biological functions of TRPV6 in PC are less fully elucidated. Therefore, we analyzed the expression of TRPV6 for clinicopathological characteristics, predicting survival in the patients, and the effect of silencing TRPV6 on apoptosis, proliferation, cell cycle, migration, invasion and chemotherapy sensitivity of PC cells, respectively, to gemcitabine, oxaliplatin and cisplatin. TRPV6 plays a promising role in the development and progression of PC. Numb was originally discovered as a determinant of cell fate (10) responsible for plenty of signal transduction pathways (Hedgehog, P53), endocytosis, cell polarity determination, and ubiquitination (11), and to play an important role in cancers. Numb was recently found as a new interacting partner with TRPV6 (12). Numb inhibits activity of TRPV6 via electrostatic interaction in breast cancer cells. Numb regulates Ca cation influx via TRPV6 (13). Ca cation influx stimulated the GSK3 $\beta$, AKT, MAPKinase pathway involved in TRPV6-specific mediating cell proliferation. Silencing Numb reduced expression of TRPV6 in prostate cancer cell lines (12). On the contrary, knockdown of Numb increased the TPRV6 expression in the breast cancer cells. On the other hand, silencing TRPV6 increased the Numb expression. Numb and TRPV6 regulate the protein stability and degradation of each other (13). Finally, we observed the interaction between TRPV6 and Numb in PC cells.

\section{Materials and methods}

Pancreatic cancer specimens. Tumor specimens and their corresponding adjacent noncancerous tissues were selected from patients who were pathologically confirmative of PC during pancreatic operation in the Shenyang Medical Center between January 2005 to January 2014. None received chemotherapy or radiotherapy in the preoperative period. The pathologic diagnosis and differentiation were confirmed by three independent pathologists by TNM classification, 7 th edition of the UICC 2010. Fresh specimens were snapfrozen in the liquid nitrogen rapidly after operation. The study was approved by the Institutional Ethics Committee of China Medical University and written informed consent was obtained from the patients.

Pancreatic cancer cell lines. We obtained BxPC-3, AsPC-1, SW1990, PANC-1 cells from the Cell Bank of the Chinese Academy of Sciences. We purchased Capan-2 cells from the 
American Type Culture Collection (ATCC). We maintained the five cell lines in growth medium supplemented with $10 \%$ fetal bovine serum and $100 \mu \mathrm{g} / \mathrm{ml}$ of penicillin and streptomycin (Hyclone, Logan, UT, USA).

RNA interference. We used small interfering RNA for TRPV6 (TRPV6-siRNA) and counterpart negative control (Neg.Cont) oligonucleotides (Shanghai GenePharma Co. Ltd.) transfected with Lipofectamine 2000 (Invitrogen, Carlsbad, CA, USA). The target sequences of oligonucleotides for the TRPV6 siRNA and Neg.Cont were as follows: TRPV6-Si1, 5'-CCAA GGAGAAAGGGCUAAUTT-3'; TRPV6-Si2, 5'-CCAUAU AUCUGCUGUACAUTT-3'; TRPV6-Si3, 5'-CUGCGUGG GAUAAUCAACATT-3'. Neg.Cont: Sense, 5'-UUCUCCGAA CGUGUCACGUTT-3'; Antisense, 5'-ACGUGACACGUU CGGAGAATT-3'.

Immunohistochemistry staining. Immunohistochemistry was performed as previously described (14). Briefly, tissue sections $(5 \mu \mathrm{m})$ were blocked with hydrogen peroxide and then incubated with mouse polyclonal anti-TRPV6 (1:400, Invitrogen), overnight at $4^{\circ} \mathrm{C}$. As a negative control, normal IgG was used as the primary antibody at the same dilution. Stained tissue sections were reviewed and scored according to Masunaga et al (15).

RNA preparation and quantitative real-time PCR. With TRIzol reagent (Takara), total RNA was extracted, and then quantitative PCR was performed with a SYBR Green II (Takara) on a Thermal Cycler Dice Real-time System (Takara) with the following protocol: $30 \mathrm{sec}$ at $95^{\circ} \mathrm{C}$ followed by two-step PCR for 40 cycles of $95^{\circ} \mathrm{C}$ for $5 \mathrm{sec}$ and $64^{\circ} \mathrm{C}$ for $30 \mathrm{sec}$. Gene expression was normalized relative to its GAPDH mRNA with the $\Delta \Delta C T$ method. The sequences of the PCR primers were as follows: TRPV6 forward, 5'-ATGGT GATGCGGCTCATCAGTG-3' and reverse, 5'-GTAGAAG TGGCCTAGCTCCTCG-3'; GAPDH forward, 5'-CTCCTC CTGTTCGACAGTCAGC-3', and reverse 5'-CCCAATAC GACCAAATCCGTT-3'.

Western blotting. Pancreatic cancer tissues and pancreatic cancer cell samples were washed with ice-cold PBS and then lysed by RIPA (Beyotime, Jiangsu, China). The lysates were separated in SDS-PAGE (10\%). Resolved protein was transferred onto a polyvinylidene difluoride (PVDF) membrane (Millipore, Bedford, MA, USA). The membranes were blocked by $5 \%$ skim milk solution in TBST buffers, and were incubated with primary antibodies for TRPV6 (1:500 Abcam, Cambridge, UK, ab63084), PCNA (1:1000, Abcam, ab29), Bax (1:1000, Abcam, ab32503), Bcl-2 (1:500, Abcam, ab692), E-cadherin (1:400, Santa Cruz Biotechnology, Santa Cruz, CA, USA, sc7870) MMP9 (1:400, Santa Cruz Biotechnology, sc6840), $\beta$-catenin (1:1500, ProteinTech Group, Chicago, IL, USA, 51067-2-AP), cyclin-D1 (1:1000, ProteinTech Group, 12363-1-AP), Numb (1:1000, Cell Signaling Technology, Danvers, MA, USA, \#8650). overnight at $4^{\circ} \mathrm{C}$. PVDF membranes were washed in TBST and incubated with horseradish peroxidase-conjugated secondary antibodies (1:10000, ProteinTech Group, SA00001-15 and SA00001-1) $2 \mathrm{~h}$ at $37^{\circ} \mathrm{C}$. Antibody against GAPDH (1:1000, Cell Signaling Technology,
Table I. TRPV6 expression with clinicopathological parameters from pancreatic cancer patients.

\begin{tabular}{|c|c|c|c|}
\hline Parameters & $\begin{array}{c}\text { TRPV6 } \\
\text { negative }\end{array}$ & $\begin{array}{l}\text { TRPV6 } \\
\text { positive }\end{array}$ & P-value \\
\hline Age (year) & & & 0.466 \\
\hline$<65$ & 20 & 31 & \\
\hline$\geq 65$ & 12 & 13 & \\
\hline Sex & & & 0.450 \\
\hline Male & 23 & 28 & \\
\hline Female & 9 & 16 & \\
\hline Size $(\mathrm{cm})$ & & & $0.024^{\mathrm{a}}$ \\
\hline$<2.5$ & 20 & 16 & \\
\hline$\geq 2.5$ & 12 & 28 & \\
\hline T Stage & & & $0.029^{\mathrm{a}}$ \\
\hline $\mathrm{T} 1+\mathrm{T} 2$ & 19 & 15 & \\
\hline $\mathrm{T} 3$ & 13 & 29 & \\
\hline Lymph node & & & 0.893 \\
\hline No & 23 & 31 & \\
\hline N1 & 9 & 13 & \\
\hline Vascular permeation & & & 0.560 \\
\hline Absent & 19 & 29 & \\
\hline Present & 13 & 15 & \\
\hline CA199 (U/ml) & & & 0.518 \\
\hline$<37$ & 8 & 14 & \\
\hline$\geq 37$ & 24 & 30 & \\
\hline Perineural invasion & & & 0.174 \\
\hline Absent & 21 & 35 & \\
\hline Present & 11 & 9 & \\
\hline Hepatic metastasis & & & 0.815 \\
\hline Absent & 21 & 30 & \\
\hline Present & 11 & 14 & \\
\hline
\end{tabular}

${ }^{\mathrm{a}} \mathrm{P}<0.05$.

\#P04406) was used as an internal control. The protein of interest was visualized using ECL Western blotting substrate (Pierce, Biotechnology, Rockford, IL, USA).

Cell Counting Kit-8 assay. When cell proliferation was detected, at an initial density of 2,000 cells/well, cells were plated in 96-well cultural dishes, Si-TRPV6 $24 \mathrm{~h}$ after transfection. Cells were incubated for $0,24,48,72 \mathrm{~h}$. CCK8 (Thiazolyl Blue) solution was added. When drug susceptibility was detected, cells had reached the density of 5,000 cells/well, $24 \mathrm{~h}$ after Si-TRPV6 transfection. Different concentrations of gemcitabine, oxaliplatin or cisplatin were added, respectively, and incubated for $48 \mathrm{~h}$.

Cell migration and invasion assays. After $48 \mathrm{~h}$ of transfection, we adjusted the density of $2 \times 10^{5}$ cells $/ \mathrm{ml}$ in every group. The upper Transwell chamber (Costar; 24-well insert, pore size: $8 \mu \mathrm{m}$ ) was filled with $200 \mu \mathrm{l}$ cell suspension, and the lower chamber was filled with $500 \mu \mathrm{l}$ of medium. For the 
A

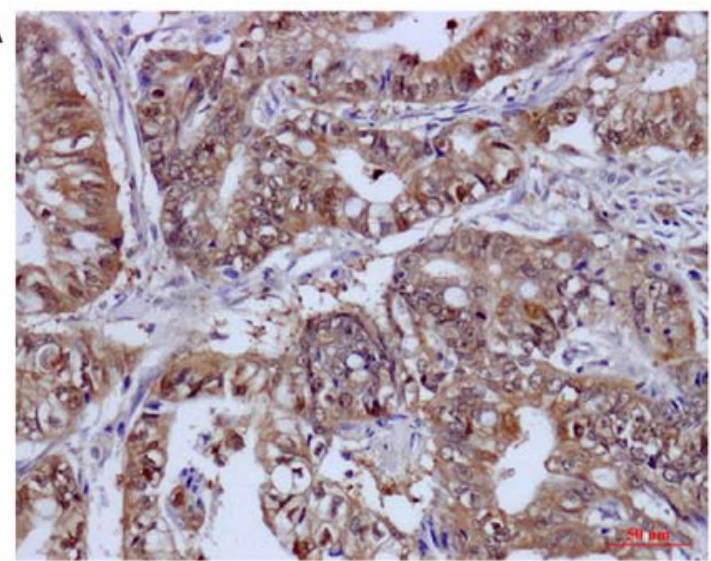

B

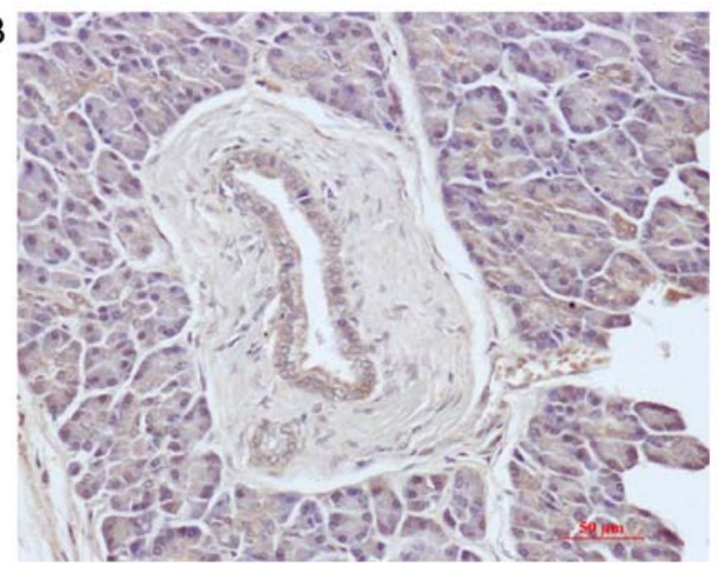

Figure 1. Representative of TRPV6 expression in PC (A) and peritumor tissue (B) detected by immunostaining using anti-TRPV6 antibody (brown). (magnification, $\mathrm{x} 200$ ).

invasion assay, polycarbonate filters coated with $50 \mu$ l Matrigel (1:9, BD Bioscience) were placed in a Transwell chamber. Cells were incubated for $24 \mathrm{~h}$ for the migration assay and $48 \mathrm{~h}$ for the invasion assay. Then, the cells on the upper surface were wiped, and the cells on the lower surface were fixed with $4 \%$ paraformaldehyde and stained with $0.1 \%$ crystal violet. The migratory cells were visualized and counted in five random visual fields per insert under an inverted microscope at x200 magnification.

Cell cycle and apoptosis. Pancreatic cancer cells were treated with Si-TRPV6 or Neg.Cont oligo. Cells with a density of $500,000 /$ well were trypsinized, and collected and stained with the Annexin V-keyFluor555 apoptosis detection kit (KeyGene, Nanjing, China). Cell cycle analysis was performed after staining with propidium iodide (Beyotime). The distribution was quantified using a flow cytometer.

Statistical analysis. Statistical analyses were performed with SPSS 14.0. The paired-sample t-test was used for TRPV6 expression in paired samples of $\mathrm{PC}$ and its normal tissue. The TRPV6 expression and clinicopathological parameters were used for the Chi-squared test. Differences in survival were assessed with Kaplan-Meier method and analyzed using the log-rank test. Cox's proportional hazards regression model was used to analyze independent prognostic factors. Cell proliferation, apoptosis, cell cycle, invasion and migration assays were expressed as means \pm SE. $\mathrm{P}<0.05$ was considered to indicate a statistically significant difference.

\section{Results}

Immunohistochemistry showed that TRPV6 protein was overexpressed in 57.9\% (44/76) in PC tissue and 19.7\% (15/76) in their matched non-tumour adjacent tissues. Specifically, TRPV6 expression was significantly increased in PC tissue in comparison to their counterpart non-tumor adjacent tissues ( $\mathrm{t}=3.039, \mathrm{P}=0.003)$. TRPV6 was mostly expressed in the cytoplasm (Fig. 1A and B).

TRPV6 was overexpressed in PC tissues, which predicted a larger size of tumor $(\mathrm{P}=0.024)$ and advanced $\mathrm{T}$ pathological stage $(\mathrm{P}=0.029)$ (Table I. Survival rate of the negative TRPV6

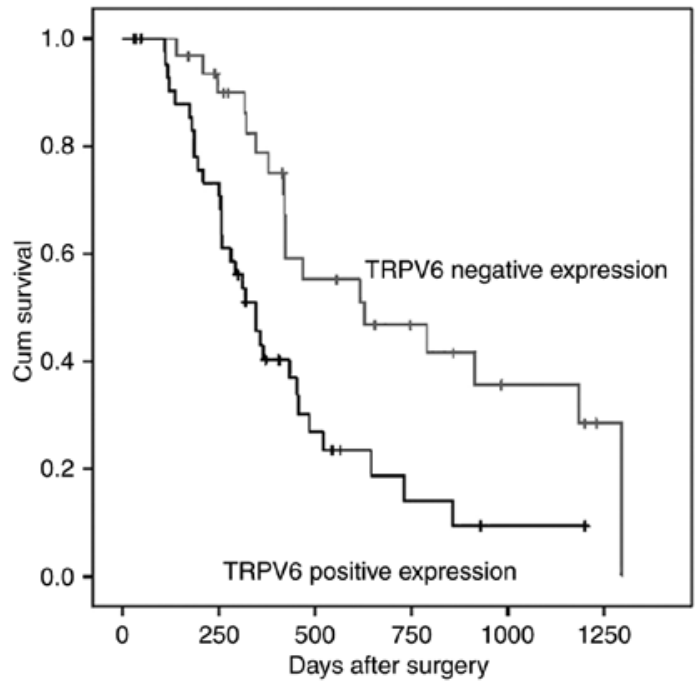

Figure 2. Cumulative Kaplan-Meier survival curve associated with TRPV6 protein in PC patients.

expression group was significantly better than that of the positive TRPV6 expression group ( $\mathrm{P}=0.003$, Fig. 2). Moreover, univariate analysis showed $\mathrm{T}$ stage $(\mathrm{P}=0.016)$, and vascular permeation $(\mathrm{P}=0.012)$ influenced patient prognosis $(\mathrm{P}=0.003$, Fig. 2) as well. Moreover, a multivariate analysis via the COX proportional hazard model was conducted to evaluate the independent prognostic value of the TRPV6 expression level. The high level of TRPV6 expression (RR 2.022, 95\% CI 1.075-3.805, $\mathrm{P}=0.029)$ and vascular infiltration (RR 1.875, 95\% CI 1.051-3.346, $\mathrm{P}=0.033$ ) were associated with a poor prognosis, independent of other clinical covariates (Table II).

In all six pairs of cases, TRPV6 mRNA and protein expression were upregulated in PC tissues in comparison to counterpart normal peritumor tissues (Fig. 3).

In the TRPV6 mRNA of PC various cell lines including BxPC-3, AsPC-1, SW1990, Capan-2 and PANC-1, the highest level of relative mRNA expression detected by qRT-PCR was in Capan-2 cells, the second highest levels in SW1990 cells and lowest levels in AsPC-1 cells. In the same five pancreatic cancer cell lines, consistent with our qRT-PCR analysis, the highest protein levels of TRPV6 expression was detected by 
Table II. Univariate and multivariate analysis of clinicopathological factors for survival in 76 pancreatic cancer patients.

\begin{tabular}{lccc}
\hline Parameters & $\begin{array}{c}\text { Median survival } \\
\text { (days) }\end{array}$ & $\begin{array}{c}\text { Univariate analysis } \\
\text { P-value (log-rank) }\end{array}$ & $\begin{array}{c}\text { Multivariate analysis } \\
(95 \% \text { CI) }\end{array}$ \\
\hline Age $(<65 / \geq 65$ year) & $365 / 454$ & 0.572 & - \\
Sex (male/female) & $421 / 345$ & 0.897 & - \\
Size $(<2.5 / \geq 2.5 \mathrm{~cm})$ & $421 / 418$ & 0.151 & - \\
T Stage (T1+T2/T3) & $520 / 345$ & $0.016^{\mathrm{a}}$ & $1.662(0.899-3.071)$ \\
Lymph node (N0/N1) & $454 / 345$ & 0.532 & - \\
Perineural invasion (absent/present) & $454 / 378$ & 0.618 & - \\
Vascular permeation (absent/present) & $615 / 345$ & $0.012^{\mathrm{a}}$ & $1.875(1.051-3.346)$ \\
Hepatic metastasis (absent/present) & $454 / 378$ & 0.361 & $0.033^{\mathrm{a}}$ \\
TRPV6 (negative/positive) & $629 / 345$ & 0.003 & $2.022(1.075-3.805)$ \\
\hline
\end{tabular}

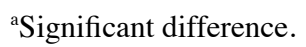
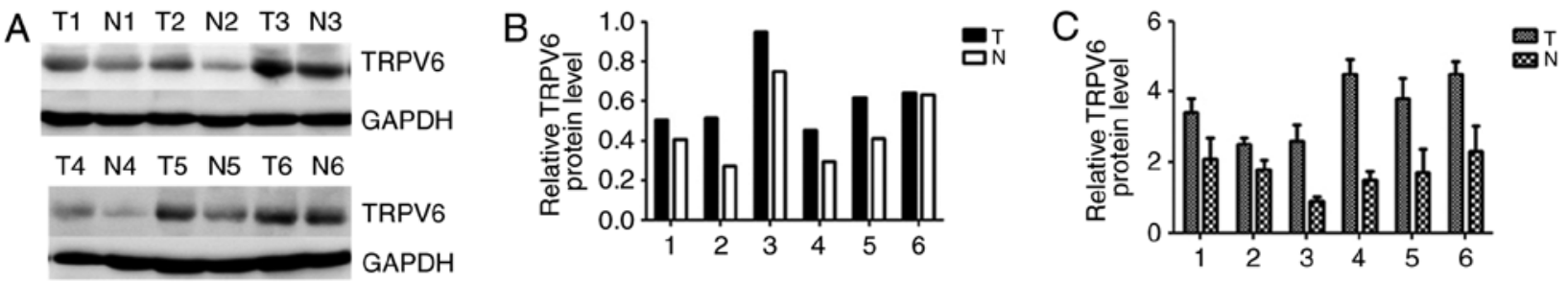

Figure 3. TRPV6 protein and mRNA in PC tissues (T) accompanying their neighboring non-tumor tissue (N). (A) Western analysis of TRPV6 protein expression in six paired cases of PC; (B and C) quantified data of TRPV6 protein and mRNA in pc tissues (T) accompany with their neighboring non-tumor tissue $(\mathrm{N})$.

western blot analysis in Capan-2 cells, the second highest levels in SW1990 cells and the lowest level was in AsPC-1 cells (Fig. 4).

We knocked down TRPV6 expression in Capan-2 cells via si-RNA transfection, and the efficiency of knockdown of the two si-RNAs was evaluated. TRPV6 mRNA expression levels of Capan-2 cells by TRPV6-si1 were reduced by $81.5 \pm 3.8 \%$ $(\mathrm{P}<0.001)$, in comparison to the negative control siRNA groups (Fig. 5A). TRPV6 protein expression levels of Capan-2 cells by TRPV6-si 2 were reduced by $63.4 \pm 5.6 \%(\mathrm{P}<0.001)$, in comparison to the negative control siRNA groups (Fig. 5B and C). The most effective TRPV6-sil was chosen for the following study.

To further examine whether TRPV6 has a definite role in $\mathrm{PC}$ progression, in vitro functional studies were conducted. TRPV6 depletion resulted in decreased proliferation both in PC cell line Capan-2 and SW1990, as determined by CCK-8 assay. Downregulation of TRPV6 expression significantly led to $34.6 \pm 2.3,34.4 \pm 2.8,37.9 \pm 1.9 \%$ decrease in Capan-2 cells $(24,48,72 \mathrm{~h})$ and $52.1 \pm 3.2,36.4 \pm 2.9,44.2 \pm 2.1 \%$ decrease in SW1990 cells $(24,48,72 \mathrm{~h})$ in the proliferation in comparison to the negative control cells (Fig. 6A and B).

We also performed cell cycle assays after si-RNA transfection using flow cytometry. The percentage of Capan-2 cells infected with si-TRPV6 in the G1 phase significantly increased from $53.26 \pm 4.52$ to $61.36 \pm 3.18 \%$, whereas the percentage of cells in the $\mathrm{S}$ phase decreased from $31.48 \pm 3.43$ to $24.56 \pm 3.10 \%$ (Fig. 7A and B). The percentage of SW1990 cells infected
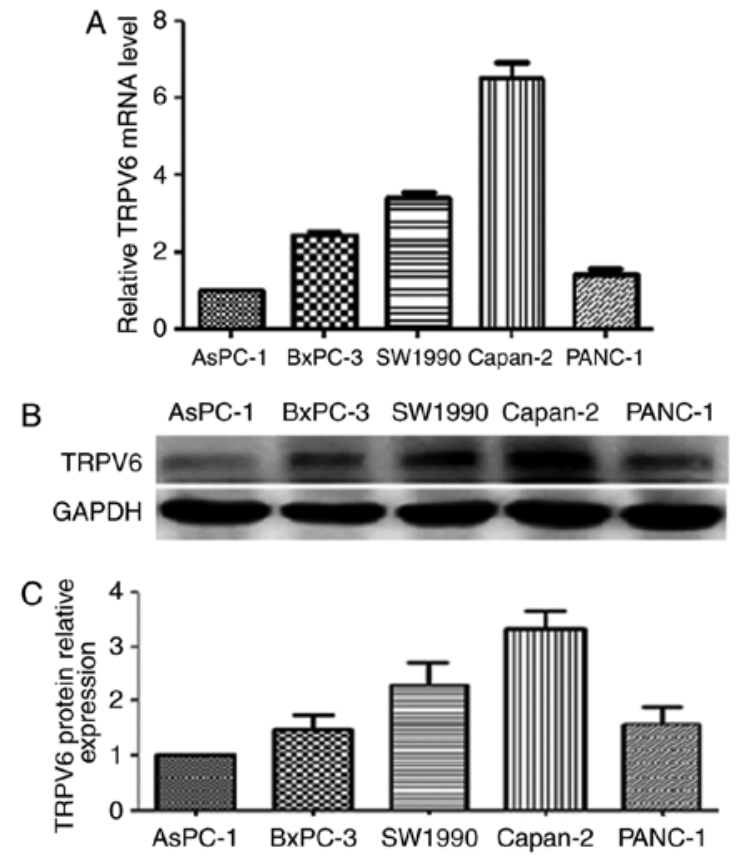

Figure 4. Expression of TRPV6 in five PC cell lines. (B) Western blotting evaluation of the expression of TRPV6 protein in five cell lines. (A and C) Quantified data of TRPV6 mRNA and protein.

with Si-TRPV6 in the G1 phase increased from $42.67 \pm 5.23$ to $55.73 \pm 3.21 \%$, whereas the percentage of cells in the $S$ phase 

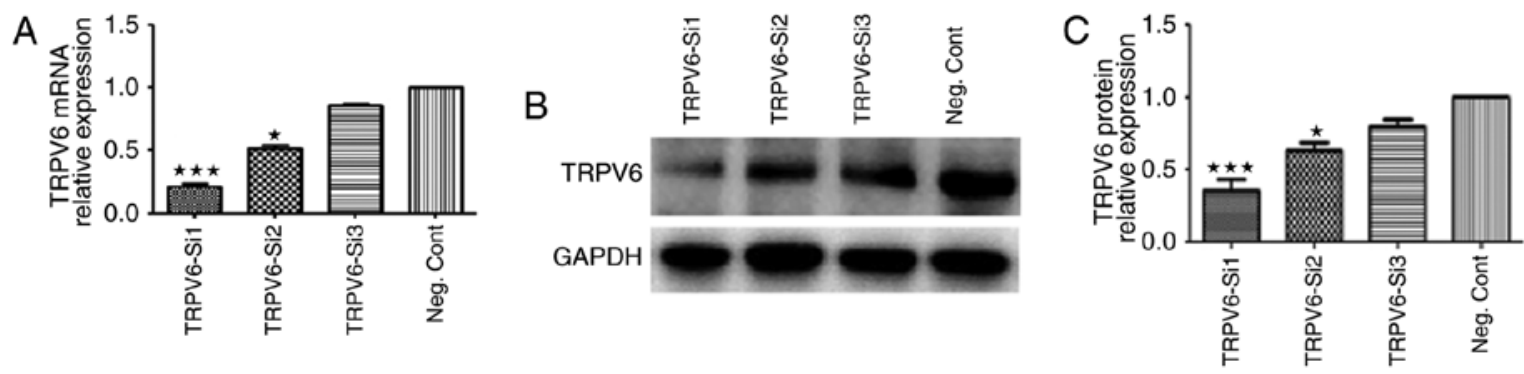

Figure 5. The infection efficiency was analyzed by qRT-PCR (A) and western blot analysis (B and C). ${ }^{*} \mathrm{P}<0.05,{ }^{* * * *} \mathrm{P}<0.001$.

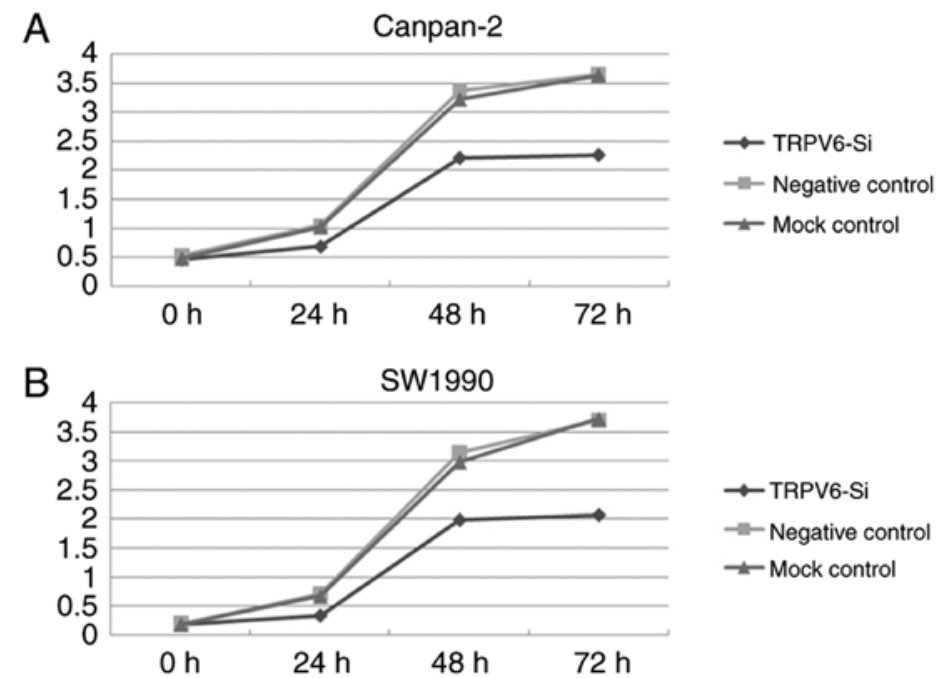

Figure 6. The CCK-8 assay detection of proliferation of Capan-2 cells (A) and SW1990 cells (B) after 24, 48 and $72 \mathrm{~h}$ of si-RNA infection.

A
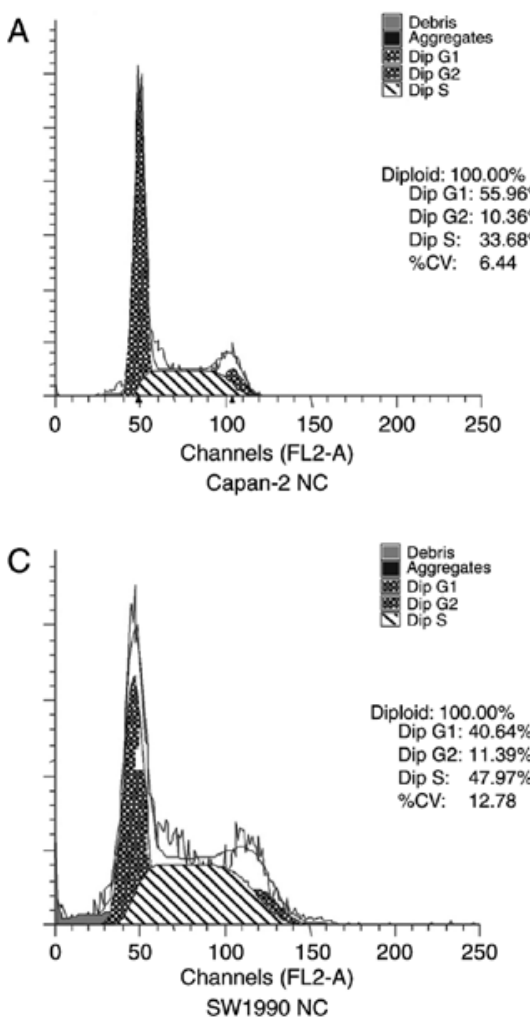

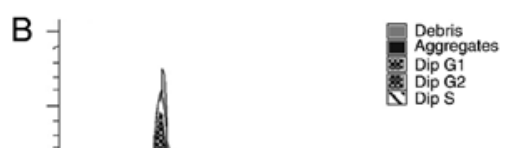

Diploid: $100.00 \%$

Dip G1: $60.06 \%$ at 60.49

Dip G2: $13.30 \%$ at 114.24

$\% \mathrm{CV}: \quad 9.92$

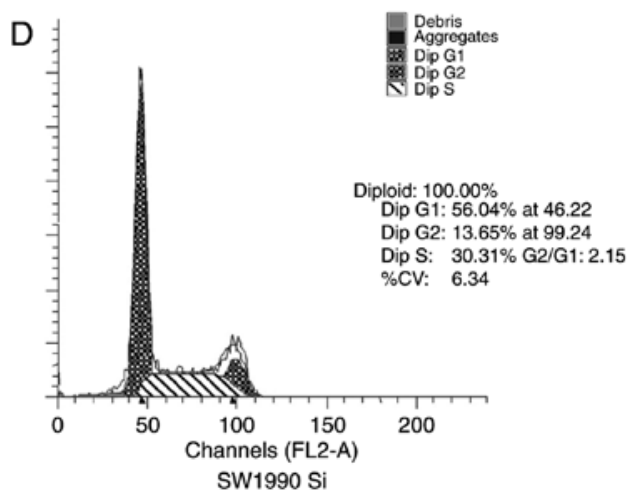

Figure 7. Cell cycle arrest by silencing of TRPV6. Proportion of Capan-2, SW1990 (A and C) cells and their TRPV6-siRNA transfected cells (B and D) in various phases of the cell cycle. NC, negative control. SI, TRPV6-siRNA. 
A

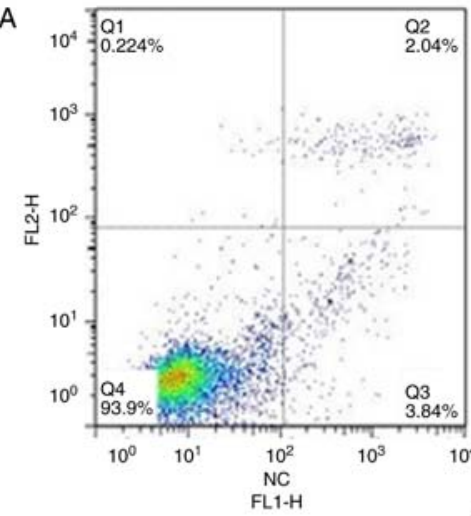

B

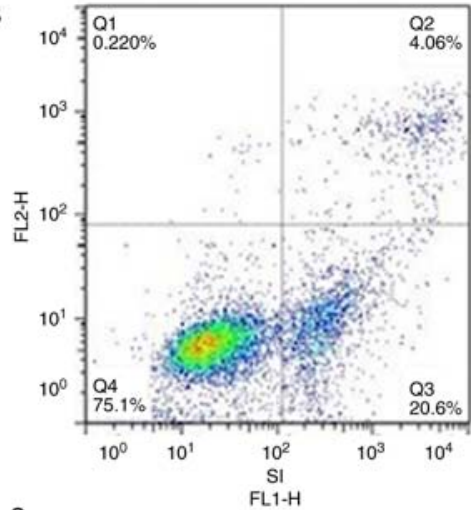

Capan-2
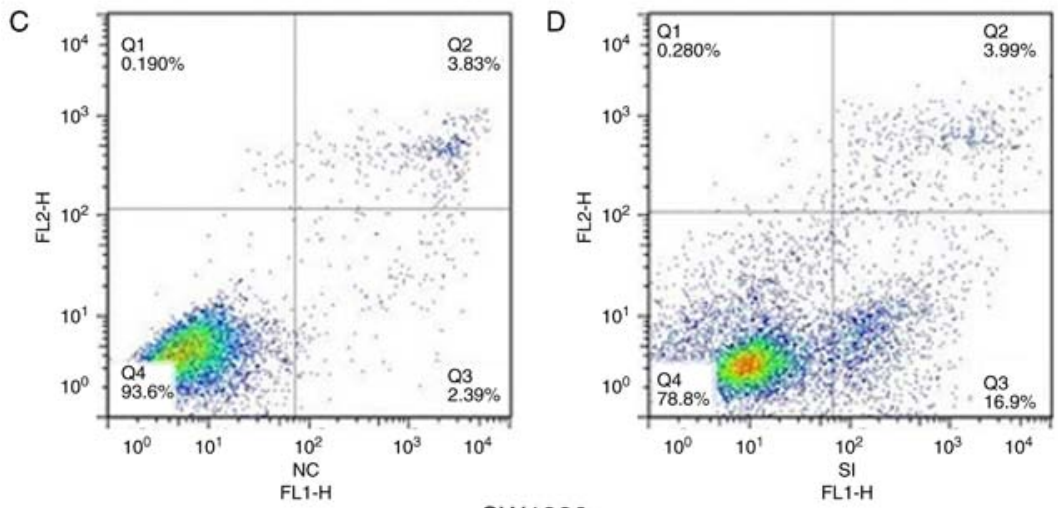

SW1990

Figure 8. Downregulation of TRPV6 promotes cell apoptosis. Capan-2 (A and B), SW1990 (C and D) cells after transfection were considered to enhance apoptosis.

decreased from $46.27 \pm 4.62$ to $30.26 \pm 2.36 \%$ (Fig. 7C and D). These results suggest that suppression of TRPV6 significantly induced G0/G1 phase arrest and promoted cell cycle progression of PC cells.

The apoptotic rate of cells infected with TRPV6-siRNA was significantly increased from $5.23 \pm 1.22$ to $23.86 \pm 2.34 \%$ in Capan-2 cells (Fig. 8A and B) and $6.18 \pm 1.87$ to $22.49 \pm 2.03 \%$ in SW1990 cells ( Fig. 8C and D), respectively, in comparison to NC cells.TRPV6-siRNA promoted apoptosis of PC cells, which in turn makes contribution towards proliferation.

The migratory cells transfected with TRPV6-siRNA were significantly decreased from $110.4 \pm 7.3$ to $45 \pm 5.4$ (Capan-2 cells) and $160 \pm 8.6$ to $70 \pm 4.8$ (SW1990 cells), respectively, in comparison to NC cells. Similarly, the invasive cells transfected with TRPV6-siRNA were also decreased from 93.8 \pm 7.5 to $50 \pm 6.8$ (Capan-2 cells) and $135.4 \pm 4.6$ to $73.7 \pm 3.9$ (SW1990 cells), respectively, in comparison to NC cells (Fig. 9). TRPV6 plays a promising role in cell invasion and migration in PC and a mechanism by which silencing TRPV6 leads to cancer metastasis inhibition in PC.

TRPV6-siRNA transfection affected the sensitivity of PC cells to gemcitabine, oxaliplatin and cisplatin. In comparison to the NC group, the inhibitory effects of oxaliplatin on cell proliferation were considerably enhanced in the si-RNA TRPV6 group. The $\mathrm{IC}_{50}$ of oxaliplatin was reduced from $15.2 \pm 1.2$ to $4.8 \pm 0.7 \mu \mathrm{g} / \mathrm{ml}(\mathrm{P}<0.05)$, the $\mathrm{IC}_{50}$ of cisplatin was reduced from $5.6 \pm 1.1$ to $3.2 \pm 0 / 9 \mu \mathrm{g} / \mathrm{ml}(\mathrm{P}>0.05)$. Gemcitabine had drug resistance, Si-TRPV6 group and NC group did not reach the cutoff of $\mathrm{IC}_{50}$ (Fig. 10).
Proteins in the cell cycle pathway and proliferation were predicted to be regulated by TRPV6 expression including PCNA in Capan-2 cells (Fig. 11A). Two core proteins Bax and $\mathrm{Bcl}-2$ in the cell apoptosis pathway were predicted to be regulated by TRPV6 expression, including in Capan-2 cells (Fig. 11A). E-cadherin and MMP9 were involved in the invasion pathway were also predicted to be regulated by TRPV6 (Fig. 11A). However, expression of $\beta$-catenin and other proteins such as cyclin-D1, cyclin-E1, CDK6, p21 (data not shown) did not change significantly. TRPV6 may regulate the cell cycle, apoptosis and invasion processes by regulating these multiple proteins.

Previously, we found the role of Numb-MDM2-P53 interaction in PC cell lines (16). In the study, Kim et al verified a novel relationship between Numb-TRPV6 in prostate cancer cells (12). Thus, we asked whether there was a novel interaction between Numb and TRPV6 in the Capan-2 cells. TRPV6-siRNA in Capan-2 cells decreased the amount of TRPV6 protein, consistent with previous results $(12,13)$ (Fig. 11A). However, knockdown of Numb expression resulted in no change of TRPV6 expression, in contrast to these results $(12,13)$ (data not shown).

\section{Discussion}

TRPV6 performed by in situ hybridization experiments by Wissenbach et al (17) were found in the normal pancreatic tissue rather than pancreatic carcinoma in only two paired samples. In this study, it was demonstrated that TRPV6 protein 
A

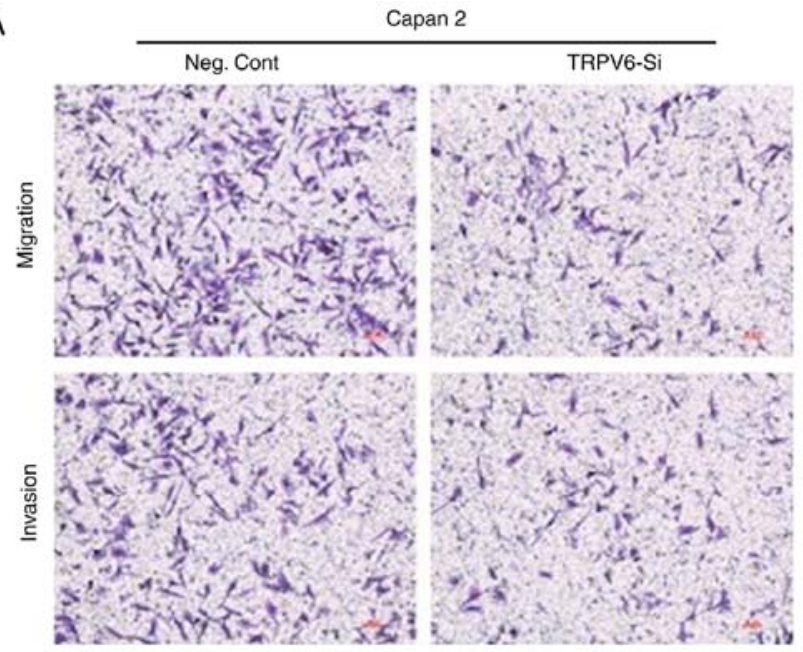

C

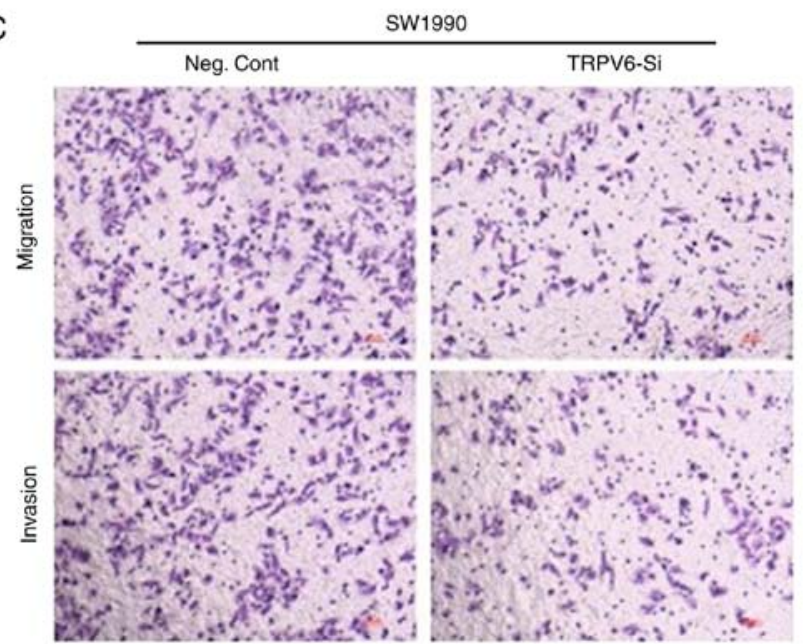

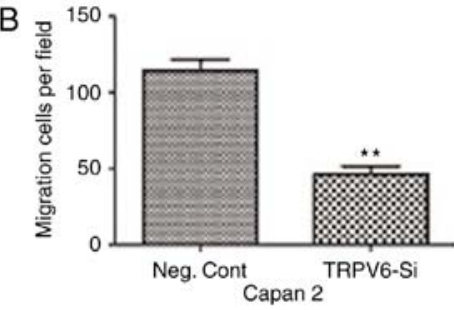
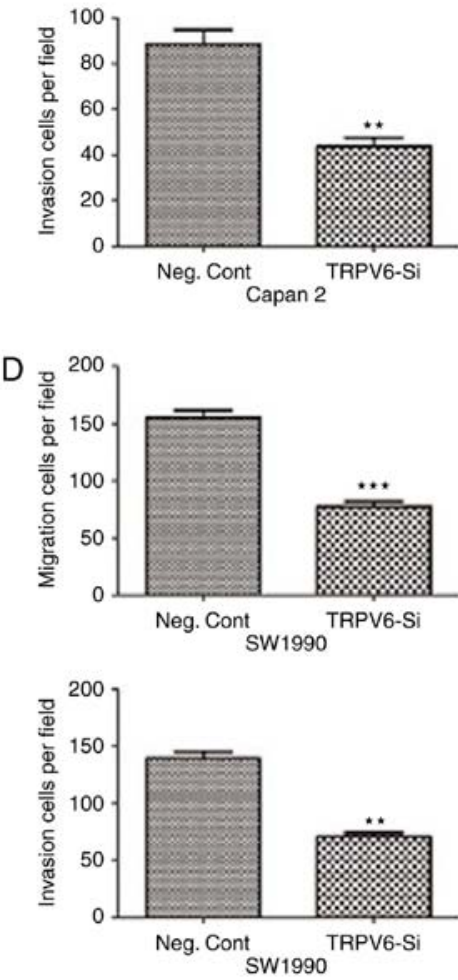

Figure 9. Downregulation of TRPV6 inhibits cell invasion and migration in the Capan-2 and SW1990 (A and C). Invasion and migration profiles of Capan-2 and SW1990 cells are depicted by histograms (B and D). ${ }^{* *} \mathrm{P}<0.01,{ }^{* * * *} \mathrm{P}<0.001$.

and mRNA level were generally upregulated in PC tissues compared to adjacent normal tissue. Positive expression of TRPV6 is significantly related with the unfavorable survival in the PC patients suggesting that TRPV6 is an oncogene in $\mathrm{PC}$ and might participate in the PC development and progression. Some reports suggested TRPV6 as important biomarkers in the development and progression of breast cancer, colon cancer, prostate cancer, thyroid cancer and parathyroid cancer $(3,5,8,17,18)$. However, decreased TRPV6 mRNA and protein level was observed from esophageal cancer, nonsmall cell lung cancer and renal cancer. The results may be related to physiological disorder caused by tumor development or non-identical modification in tumorigenic cells. TRPV6 regulated by various upstream genes may result in tumor suppressive or oncogenic function in different cancers. Some studies showed that TRPV6 may play a protective role in colon cancer and gastric cancer $(3,19,20)$. Curcumin could up-regulate TRPV6 to promote calcium uptake for the protection against colon cancer (19). The abundance of TRPV6 could determine its protective role via capsaicin against gastric cancer (20). Thus, antitumor effect of TRPV6 regulated by some genes may be associated with the protective role in some cancers. On the other hand, oncogenic effect of TRPV6 could be regulated by different genes in other cancers. The pleiotropic

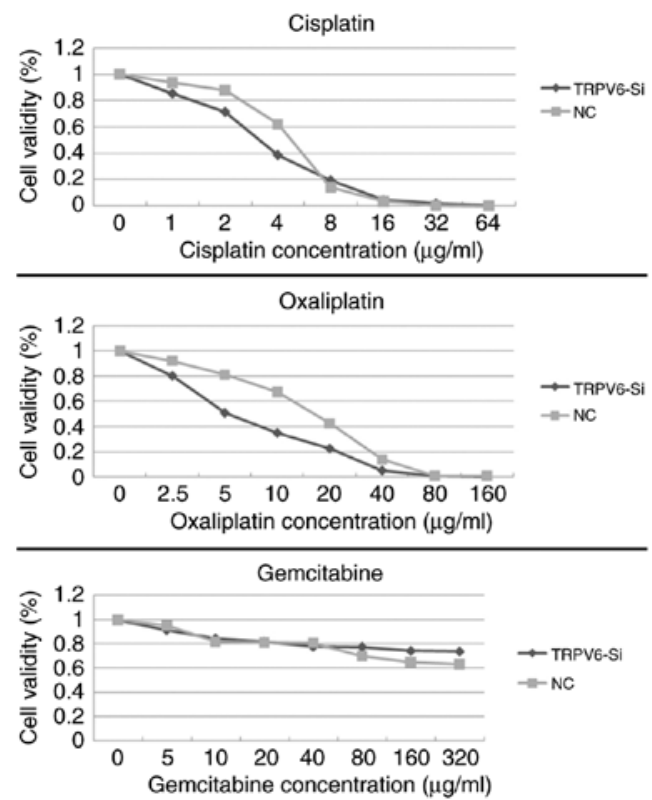

Figure 10. The $\mathrm{IC}_{50}$ change in Capan-2 after TRPV6-siRNA transfection. After chemotherapy with gemcitabine, oxaliplatin, and cisplatin, cytotoxicity assay is shown by CCK8. Transfection with TRPV6-siRNA could significantly decrease the $\mathrm{IC}_{50}$ of oxaliplatin, but had little effect on gemcitabine and cisplatin. 

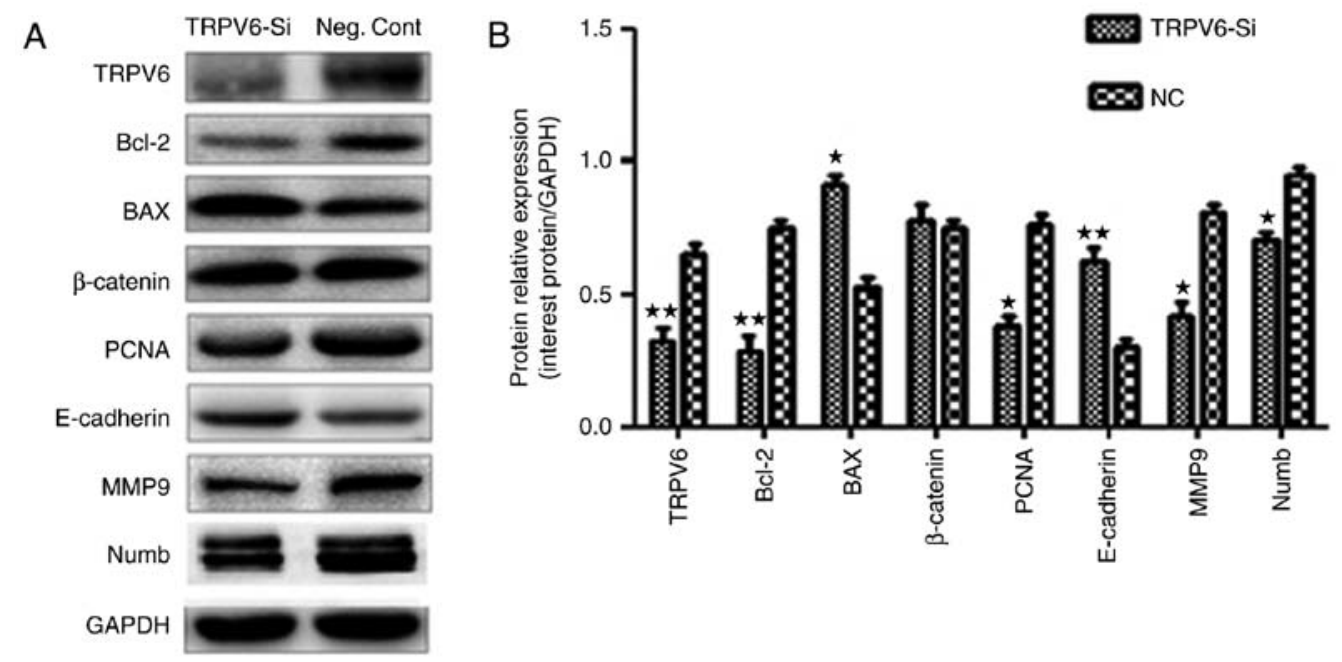

Figure 11. siRNA targeting TRPV6 is potent on the expression of cell cycle, apoptosis, invasion-related genes and Numb (A). Representative histograms depicting related gene expression after TRPV6-siRNA in the Capan-2 cells (B). ${ }^{*} \mathrm{P}<0.05,{ }^{* * *} \mathrm{P}<0.01$.

functions of TRPV6 and its mechanism should be clarified according to distinct cellular context. TRVP6 is a highly selective calcium channel contributing to store-operated calcium entry (SOCE) activity involving the plasma membrane via the Orai1/TRPC1-mediated $\mathrm{Ca}^{2+} /$ Annexin I/S100 pathway in prostate cancer cell line (21). The role of calcium are known to affect proliferation (22), apoptosis (23) and migration (24). Peleg et al found silencing TRPV6 inhibited proliferation and induced apoptosis in colon carcinoma cells (25). On the other hand, Schwarz et al found overexpression of TRPV6 increased proliferation of HEK-293 cells in a $\mathrm{Ca}^{2+}$ dependent manner (22). Lehen'kyi et al regarded that TRPV6-siRNA was directly involved in proliferation and cell cycle in $\mathrm{LNCaP}$ cells (26). In our study, using Capan-2 and SW1990 PC cell transfected with TRPV6-siRNA, cell proliferation, migration and invasion was suppressed; the cell cycle was arrested in the G1 phase; and apoptosis was promoted. Overexpression of TRPV6 in PC may alter key features of the cells leading to malignant biological behavior.

At present, gemcitabine is the first line chemotherapy of pancreatic cancer. There are no randomized data favoring neoadjuvant (including 5-fluorouracil, irinotecan and oxaliplatin, gemcitabine with or without abraxane, or docetaxel and capecitabine) overwhelming adjuvant therapy (27). Docetaxel/ oxaliplatin (DocOx) combination as the second line treatment for advanced pancreatic cancer is an effective option (28). In our study, silencing TRPV6 expression can significantly increase chemotherapy sensitivity of PC cells to oxaliplatin, rather than gemcitabine. It could provide a proper research basis on the drug related to calcium decreasing the side effect of oxaliplatin for pancreatic cancer.

PCNA has been found to participate in cell cycle regulation and DNA replication. Our study showed TRPV6-siRNA could decrease the expression of PCNA, consistent with the study of Lehen'kyi et al (26). Although proteins such as cyclin-D1, cyclin-E1, CDK6, p21 did not change significantly, there would be some genes related to cell cycle such as cyclin-A, cyclin-B1 to be detected in the future. Maybe phosphorylation or other modifications occurred in the protein such as CDK6 instead of alteration of amount of protein. The further experiments should be validated. Increasing Bax/Bcl-2 ratios by TRPV6siRNA, apoptosis was promoted suggesting a mitochondrial apoptotic pathway $(29,30)$. Functionally, E-cadherin acts as the cancer suppressor gene and regulates cell polarity, differentiation, migration and invasion (31). Invasion and metastasis of tumor involving degradation of ECM (extracellular matrix) and basement membrane by MMP-9 (matrix metalloproteinase-9) are critical determinants of cancer morbidity (32). It is probable that upregulation of E-cadherin and downregulation by TRPV6-siRNA could be involved in invasion and migration of PC.

We showed that TRPV6 reduced expression of Numb protein in Capan-2 cells indicating Numb probably acting as a oncogene. Knockdown of Numb did not alter TRPV6 expression in PC cell lines. It was very confusing that our previous study showed Numb as the cancer suppressor gene (33). It is hypothesized that regulatory factors in TRPV6 toward Numb functions abnormally in PC cells due to unknown mutations.

In summary, TRPV6 was significantly overexpressed in PC tissues and cell lines. This could indicate a promising role of TRPV6 in PC carcinogenesis. Moreover, TRPV6 had significant correlation with the cell cycle, apoptosis and metastasis pathways and regulated expression of related proteins. Inhibiting TRPV6 expression can increase chemotherapy sensitivity of PC cells to the second line drug oxaliplatin. Our study indicted the feasibility of TRPV6 as a potential therapeutic for PC.

\section{Acknowledgements}

The study was sponsored by Chinese National Science Foundation (no. 81672835) to M.D.

\section{References}

1. Ansari D, Tingstedt B, Andersson B, Holmquist F, Sturesson C, Williamsson C, Sasor A, Borg D, Bauden M and Andersson R: Pancreatic cancer: Yesterday, today and tomorrow. Future Oncol 12: 1929-1946, 2016. 
2. Chari ST, Kelly K, Hollingsworth MA, Thayer SP, Ahlquist DA, Andersen DK, Batra SK, Brentnall TA, Canto M, Cleeter DF, et al: Early detection of sporadic pancreatic cancer: Summative review. Pancreas 44: 693-712, 2015.

3. Lehen'kyi V, Raphaël M and Prevarskaya N: The role of the TRPV6 channel in cancer. J Physiol 590: 1369-1376, 2012.

4. Wissenbach U, Niemeyer B, Himmerkus N, Fixemer T, Bonkhoff $\mathrm{H}$ and Flockerzi V: TRPV6 and prostate cancer: Cancer growth beyond the prostate correlates with increased TRPV6 $\mathrm{Ca}^{2+}$ channel expression. Biochem Biophys Res Commun 322: 1359-1363, 2004.

5. Giusti L, Cetani F, Da Valle Y, Pardi E, Ciregia F, Donadio E, Gargini C, Piano I, Borsari S, Jaber A, et al: First evidence of TRPV5 and TRPV6 channels in human parathyroid glands: Possible involvement in neoplastic transformation. J Cell Mol Med 18: 1944-1952, 2014.

6. Ouadid-Ahidouch H, Dhennin-Duthille I, Gautier M, Sevestre H and Ahidouch A: TRP calcium channel and breast cancer: Expression, role and correlation with clinical parameters. Bull Cancer 99: 655-664, 2012 (In French).

7. Zhang SS, Xie X, Wen J, Luo KJ, Liu QW, Yang H, Hu Y and $\mathrm{Fu}$ JH: TRPV6 plays a new role in predicting survival of patients with esophageal squamous cell carcinoma. Diagn Pathol 11: 14, 2016.

8. Fan H, Shen YX and Yuan YF: Expression and prognostic roles of TRPV5 and TRPV6 in non-small cell lung cancer after curative resection. Asian Pac J Cancer Prev 15: 2559-2563, 2014

9. Wu Y, Miyamoto T, Li K, Nakagomi H, Sawada N, Kira S, Kobayashi H, Zakohji H, Tsuchida T, Fukazawa M, et al: Decreased expression of the epithelial $\mathrm{Ca}^{2+}$ channel TRPV5 and TRPV6 in human renal cell carcinoma associated with vitamin D receptor. J Urol 186: 2419-2425, 2011

10. Uemura T, Shepherd S, Ackerman L, Jan LY and Jan YN: numb, a gene required in determination of cell fate during sensory organ formation in Drosophila embryos. Cell 58: 349-360, 1989.

11. Gulino A, Di Marcotullio L and Screpanti I: The multiple functions of Numb. Exp Cell Res 316: 900-906, 2010.

12. Kim SY, Hong C, Wie J, Kim E, Kim BJ, Ha K, Cho NH, Kim IG, Jeon JH and So I: Reciprocal positive regulation between TRPV6 and NUMB in PTEN-deficient prostate cancer cells. Biochem Biophys Res Commun 447: 192-196, 2014.

13. Kim SY, Yang D, Myeong J, Ha K, Kim SH, Park EJ, Kim IG Cho NH, Lee KP, Jeon JH, et al: Regulation of calcium influx and signaling pathway in cancer cells via TRPV6-Numbl interaction. Cell Calcium 53: 102-111, 2013.

14. Yamasawa K, Nio Y, Dong M, Yamaguchi K and Itakura M: Clinicopathological significance of abnormalities in Gadd45 expression and its relationship to p53 in human pancreatic cancer. Clin Cancer Res 8: 2563-2569, 2002.

15. Masunaga R, Kohno H, Dhar DK, Ohno S, Shibakita M Kinugasa S, Yoshimura $\mathrm{H}$, Tachibana $\mathrm{M}$, Kubota $\mathrm{H}$ and Nagasue N: Cyclooxygenase-2 expression correlates with tumor neovascularization and prognosis in human colorectal carcinoma patients. Clin Cancer Res 6: 4064-4068, 2000.

16. Sheng W, Dong M, Zhou J, Li X, Liu Q, Dong Q and Li F: The relationship and clinicopathological significance of Numb, MDM2 and $\mathrm{p} 53$ expression in human pancreatic cancer. Zhonghua Wai Ke Za Zhi 52: 675-681, 2014 (In Chinese).

17. Wissenbach U, Niemeyer BA, Fixemer T, Schneidewind A Trost C, Cavalie A, Reus K, Meese E, Bonkhoff $\mathrm{H}$ and Flockerzi V: Expression of CaT-like, a novel calcium-selective channel, correlates with the malignancy of prostate cancer. J Biol Chem 276: 19461-19468, 2001.

18. Dhennin-Duthille I, Gautier M, Faouzi M, Guilbert A, Brevet M, Vaudry D, Ahidouch A, Sevestre $\mathrm{H}$ and Ouadid-Ahidouch $\mathrm{H}$ High expression of transient receptor potential channels in human breast cancer epithelial cells and tissues: Correlation with pathological parameters. Cell Physiol Biochem 28: 813-822, 2011.
19. Bartik L, Whitfield GK, Kaczmarska M, Lowmiller CL, MoffetEW, Furmick JK, HernandezZ, Haussler CA, Haussler MR and Jurutka PW: Curcumin: A novel nutritionally derived ligand of the vitamin D receptor with implications for colon cancer chemoprevention. J Nutr Biochem 21: 1153-1161, 2010.

20. Chow J, Norng M, Zhang J and Chai J: TRPV6 mediates capsaicin-induced apoptosis in gastric cancer cells - Mechanisms behind a possible new 'hot' cancer treatment. Biochim Biophys Acta 1773: 565-576, 2007

21. Raphaël M, Lehen'kyi V, Vandenberghe M, Beck B, Khalimonchyk S, Vanden Abeele F, Farsetti L, Germain E, Bokhobza A, Mihalache A, et al: TRPV6 calcium channel translocates to the plasma membrane via Orail-mediated mechanism and controls cancer cell survival. Proc Natl Acad Sci USA 111: E3870-E3879, 2014.

22. Schwarz EC, Wissenbach U, Niemeyer BA, Strauss B, Philipp SE, Flockerzi V and Hoth M: TRPV6 potentiates calcium-dependent cell proliferation. Cell Calcium 39: 163-173, 2006.

23. Vanden Abeele F, Roudbaraki M, Shuba Y, Skryma R and Prevarskaya N: Store-operated $\mathrm{Ca}^{2+}$ current in prostate cancer epithelial cells. Role of endogenous $\mathrm{Ca}^{2+}$ transporter type 1 . J Biol Chem 278: 15381-15389, 2003.

24. Tauzin S, Chaigne-Delalande B, Selva E, Khadra N, Daburon S, Contin-Bordes C, Blanco P, Le Seyec J, Ducret T, Counillon L, et al: The naturally processed CD95L elicits a c-yes/calcium/ PI3K-driven cell migration pathway. PLoS Biol 9: e1001090, 2011.

25. Peleg S, Sellin JH, Wang Y, Freeman MR and Umar S: Suppression of aberrant transient receptor potential cation channel, subfamily V, member 6 expression in hyperproliferative colonic crypts by dietary calcium. Am J Physiol Gastrointest Liver Physiol 299: G593-G601, 2010.

26. Lehen'kyi V, Flourakis M, Skryma R and Prevarskaya N: TRPV6 channel controls prostate cancer cell proliferation via $\mathrm{Ca}(2+)$ NFAT-dependent pathways. Oncogene 26: 7380-7385, 2007.

27. Russo S, Ammori J, Eads J and Dorth J: The role of neoadjuvant therapy in pancreatic cancer: A review. Future Oncol 12: 669-685, 2016.

28. Ettrich TJ, Perkhofer L, von Wichert G, Gress TM, Michl P, Hebart HF, Büchner-Steudel P, Geissler M, Muche R, Danner B, et al: DocOx (AIO-PK0106): A phase II trial of docetaxel and oxaliplatin as a second line systemic therapy in patients with advanced pancreatic ductal adenocarcinoma. BMC Cancer 16: 21, 2016.

29. Rajan S, Choi M, Nguyen QT, Ye H, Liu W, Toh HT, Kang C, Kamariah N, Li C, Huang H, et al: Structural transition in Bcl-xL and its potential association with mitochondrial calcium ion transport. Sci Rep 5: 10609, 2015.

30. Luna-Vargas MP and Chipuk JE: The deadly landscape of proapoptotic BCL-2 proteins in the outer mitochondrial membrane. FEBS J 283: 2676-2689, 2016.

31. Serrano-Gomez SJ, Maziveyi M and Alahari SK: Regulation of epithelial-mesenchymal transition through epigenetic and posttranslational modifications. Mol Cancer 15: 18, 2016.

32. Verma S, Kesh K, Gupta A and Swarnakar S: An overview of matrix metalloproteinase 9 polymorphism and gastric cancer risk. Asian Pac J Cancer Prev 16: 7393-7400, 2015.

33. Sheng W, Dong M, Zhou J, Li X, Liu Q, Dong Q and Li F. Cooperation among Numb, MDM2 and p53 in the development and progression of pancreatic cancer. Cell Tissue Res 354: 521-532, 2013. 